\title{
INVITATION TO THE HUMAN GAIT ANALYSIS - REHABILITATION ENGINEERING OF THE GAIT -
}

Yoshihiro Ehara

\section{The Kanagawa Rehabilitation Center}

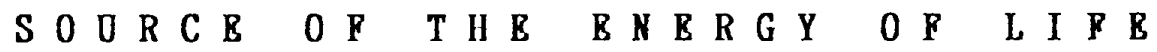

Ten billion years ago, our universe was created with big bang, and several nuclei and particles were scattered out. Nucleosynthesis happened to make further several new particles with the scattered nuclei. At the same time, coarsenes and fineness of the density of the universe were produced by the gravitational force and the repulsive force of these elementary particles. Five and a half billion years later, within high density area of the solar system, the third planet was molded. At that time, exist rate of major elements in the proto-solar system were, being that of the silicon 100 ,

\begin{tabular}{|c|c|c|c|}
\hline hydrogen & $3.18 \times 10^{6}$ & argon & 11.72, \\
\hline helium & $2.21 \times 10^{5}$ & aluminium & 8.5 \\
\hline oxygen & $2.15 \times 10^{3}$ & calcium & 7.21, \\
\hline carbon & $1.18 \times 10^{3}$ & natrium & 6.0 \\
\hline nitrogen & 374.0 & nickel & 4.8 \\
\hline neon & 344.0 & chromium & 1.27, \\
\hline magnesium & 106.0 & phosphorus & 0.96 \\
\hline silicon & 100.0 & manganese & 0.93 \\
\hline ferrum & 83.0 , & chlorine & 0.57, \\
\hline sulfur & 50.0, & kalium & 0.42. \\
\hline
\end{tabular}

The third planet was covered with rocks made of silicon and condensed oxygen and aluminium. Some other elements existed in the form of molecules or compounds or crystals. Atmosphere came into existence in the form of the mixture of methane, ammonia, hydrogen, water vapor, carbon compound, nitrogen compound and so forth on the planet being captured by the gravitational force. Water vapor within the atmosphere poured down as heavy rain to make the sea over lower ground. The planet had adequate temperature to maintain the atmosphere as gas and to keep the water as liquid. Melted metal compounds in rocks went into the sea to make salt. Some day, some elements absorbed energy and formed themselves into organic compound in which carbon atoms were their skeleton. Some day, they absorbed further energy and prepared to become more complex compound, the protein. Solar energy, high pressure or big electromagnetic force could be the contributing factor. Or the energy might came from collisions of meteorites to the planet. In a tremendous long long run, the protein became to be the coacervate. Again after a long long time, tiny but 
very complex material were produced which absorbed various materials from outer world, digested it and did autoreproduction, the LIPE was bone. It was seven billion years since the big bang.

Though over 100 elements exist on our planet, when we count the elements which are required for the constitution of the life, it is only 19. Any cell of the life, from unicellular microbiology to higher animals such as mammals, contains almost same ratio of the elements. The ratio of those elements, in weight, are

\begin{tabular}{|c|c|}
\hline oxygen & 65 \\
\hline carbon & 18 \\
\hline hydrogen & 10 \\
\hline nitrogen & 3 \\
\hline calcium & i. 5 \\
\hline phosphorus & 1 \\
\hline
\end{tabular}

We will find out that the life was created from the materials which are very easily obtainable on the planet. This fact might be related with the probability of the birth of the life.

The initial life went through hardships and gradually evolved themselves into plants. Plants are, roughly speaking, the life which can transform the solar energy into their own energy which can be freely controllable (free energy). This transformation is called the photosynthesis which makes for example sugar such as glucose from carbon dioxide and water under the exposure of sunlight. Sketchy description of this procedure in chemical equation is

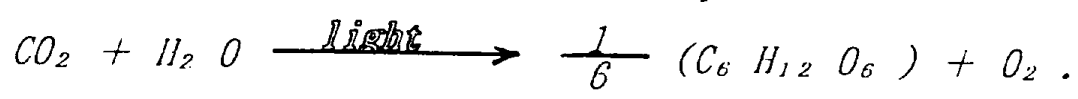

Seeing the change of the free energy, right hand term has 100 kcal per a mol bigger energy than the left hand term, this shall not happen in a general condition but using the energy of the light.

In recent research, this transformation was separated into two stage, in the former (so called light reaction) cholorophyll produces the high energy material ATP (adenosine triphosphate) with the light, in the latter (so called dark reaction) the hexose such as glucose is produced from carbon dioxide by the energy which is discharged when the ATP goes through hydrolysis.

The energy produced by plants is absorbed by herbivorous animals. The herbivorous animals eat the energy transformated by the plants in the form of cellulose, starch or vegetable protein, and re-synthesize them into glycogen, fat, protein and energy. Some bacterias in their digestive tracts help this energy re-synthes is.

The carnivorous animals eat the energy synthesized by the herbivorous animals. In this sense, animals are the life who re-synthesize their own free energy from 
the energy produced by the plants or other animals.

The life evolved so many times in a long long time, and finally the man-kind was born 99.9 billion years after the big bang. The energy sources of the human are sugar, fat and protein.

Sugar and starch (starch is the stored sugar for the plant, and glycogen is the stored sugar for the animal) are absorbed through the bowel in the form of mainly glucose into blood. Excessive glucose is stored in liver or muscle in the form of glycogen or fat. When it is required, it can be transformed easily into glucose, and is utilized. This procedure is called glycogenolysis. Usually, about $100 \mathrm{~g}$ to $200 \mathrm{~g}$ of glycogen is stored in the liver, but this amount of the glycogen is only enough for the 24 hours of demand for our body. Additional $400 \mathrm{~g}$ of glycogen is stored in the skeletal muscles, which is consumed when it is needed and resupplied from blood sugar after the activity.

Excessive fat, which will not used soon to produce heat or energy, are stored in the fat tissue as the neutral fat. Because fat has two times of heat capacity per unit weight compared with sugar or protein, it is convenient for storing. Glycogen, which is the stored sugar, are stored only in liver and muscles, but much fat are stored in the fat cell under subcutaneous tissue, in the cavum abdominis and between muscles, and so the animals can gain the body weight twice with the fat. Excessive sugar can be stored in the fat tissue processed through some chemical reactions.

Protein is digested into $20 \mathrm{kinds}$ of amino acid and is absorbed to be stored in our body and to be taken out when it is required for the growth and the repair of the body cell.

In this way, absorbed energy sources are stored in various forms, and which discharge energy with oxidation when it is required for muscle activities and so on, but this energy does not directly cause the muscle contraction. Energy produced by oxidation is used for the synthesis of ATP. When the muscle contract, ATP stored in the muscle will be used first. The muscle contract when ATP is transformed into ADP (adenosine diphosphate) through hydrolysis. Here the density of ATP seldom reduce below certain level because phosphorus is backed up from creatine phosphate and resynthesis of ATP from ADP goes quite quickly. When the density of ATP is reduced, the muscle shall be in unreversible rigor state. However amount of the creatine phosphate in muscle are so limited that it will be ended within ten seconds if further energy supply are not available. This reaction needs no oxygen at all.

In some seconds later, glycolysis reaction begins supplying the energy. It is a kind of slow starter reaction than creatine phosphate. In this reaction 2 mols of ATP are synthesized from $1 \mathrm{~mol}$ of glucose, producing pyruvic acid so lactic acid are accumulated at the end. Excessive lactic acid makes the muscle much acidic 
which stops further glycolysis. So, the energy generation with the glycolysis will be stopped within around 40 seconds if further energy supply is not available. Again, no oxygen is required in this reaction. llere, efficiency of this synthesis is $3 \%$ when we think energy of glucose (675 kcal/mol) as input and energy of ATP $((11.5 \mathrm{kcal} / \mathrm{mol}) \times 2)$ as output.

In some minutes later, oxidation starts with their effect. The pyruvic acid produced by the glycolysis reaction will produce 38 mols of ATP when they go through Krebs cycle and combined with oxygen to put out carbon dioxide and water behind. Here, efficiency of $\Lambda T P$ synthes is is $65 \%(11.5 \mathrm{kcal} \times 38 / 675 \mathrm{kcal})$. At this time accumulated lactic acid is oxidized mainly in the liver. Or, excessive lactic acid which muscle can not handle is put out into blood, reaches to the liver, and is resynthesized into glycogen again. When muscle acts so hard, lactic acid is gradually accumulating in the muscle because oxidation can not catch up with it. This is the cause of the muscle fatigue.

Now we can see that $\Lambda$ TP takes an important role in muscle contraction. One of the reason why they do not use glucose energy directly for the contraction, is that oxidation of glucose might be a kind of explosion which is difficult to be adjusted and controlled. Instead, they use easily controllable energy form which is ATP, and they disperse 35\% energy (100-65\%) as heat.

It was inevitable to obtain freely controllable ATP energy for the evolution of a life to become higher form. issential material of ATP is phosphorus and which consists of only $1 \%$ of body weight, and also very rare among our universe. It is very interesting that this rare material plays an active part as the energy source of life.

In addition to the freely controllable energy, the general operational headquarter to handle the energy was essential for life to develop themselves into higher life. The unicellular microbiology, which moves responding to the stimulation from external world and takes the outer material and digests it if they can be digested, evolved into higher form of 1 ife, which has WILL to move and hunt for their food. This creature, that is mankind, began to want to know about their origin as a life and the origin of their will. This desire yielded the science. Regret to say, it will take so many years till we can understand the mechanism of our "will".

llowever, knowledge about the locomotion, which is thought to be semi-automatic procedure, have been getting clearer gradually, for instance where the excitement occur in the brain, where the stimulation goes through, how the locomotion happen and how the outer space feedback is used for the control of the movement. The latest knowledge of this kind will be shown at the seminar.

Anyway part of "will" goes through nerve, reach muscle, and at the very end calcium is discharged into the muscle. The muscle contract with this stimulation 
utilizing nearby ATP energy. In other words, chemical bond energy is transformed into mechanical energy and heat energy. Calcium is important material not only as the bone components but also as the transmitter of the neurological stimulus.

The dynamic characteristic of muscle is affected by the amount of stimuli and also by the load. The smaller the load, the bigger the contraction speed is. When the load is so big, the speed might be zero, on in some case, the muscle might be lengthened. As the consumption energy of ATP depends on the load and the speed, muscle efficiency as the power source depends on the dynamic condition of the muscle. Therefore we should look at the dynamic muscle condition when we want to think about the efficiency of the locomotion.

The mechanical energy produced by muscle is conveyed to the body segments to which the muscle attaches. In this way muscle activities increase or some instances reduce the body mechanical energy. However, remember that the muscle activity itself cannot cause the change of location of the center of gravity of the whole body. Gravitational and floor forces are essential to do that. The body can contact to the floor in presence of the gravitational force. We can use horizontal friction force owing to this contact. Owing to this horizontal friction, we can transform internal mechanical energy into external mechanical energy which is the source of the propulsion of the center of gravity of the whole body. Thanks to the gravitation and the floor reaction forces which realize our locomotion. Conversely speaking, the life who want to move over the ground evolved on condition that the gravitation and the floor force exist.

In Greece age Aristoteles (384 322BC) told that the locomoting animals can change their position by pushing down the ground, and that if there is no resistance from the ground locomotion will not be possible.

Now, the highest creature on the third planet have established their own locomotion style, erect bipedal locomotion. Upright standing made our hands free, and made our gait efficient, but on the other hand we become slow runner and bad hunter. So, the creature developed hunting tools and group hunting tactics. These might have promoted their social system.

Gravity is well utilized especially in bipedal locomotion. He transform gravitational potential energy into the rotational energy of the whole body around the support foot and the forward progressional energy of the center of gravity of the whole body. Then reduced potential energy are supplemented with the muscle energy, and these procedures are repeated successively.

In 1682 Borelles told in his book "De Motu Animalium" that the walking is the movement to recover their upperbody balance. He established the basement of so called propulsive restrain theory. In 1836 weber brothers advocated that the walking is the falling down which is supported by the rhythmic motion of the lower legs. The essence of the bipedal locomotion is in its instability in which the 
gravitational energy are utilized well. We would not walk unstably under the abnormal control of the central nervous system or abnormal motion of the muscle. In such case we can walk only in the stable condition in which the gravitation would not be utilized well. In 1953 Saunders et al considered that well utilization of the gravitation is reflected in the smoothness of the center of gravity pass during walking, and told that main factors of this are horizontal rotation of the pelvis, frontal rotation of the pelvis, slight bending of the knee during the support phase and the ankle function.

Here, there will be so many ways of motion of the joints to make the center of gravity pass very smoothly. However, as a fact when we look at the normal gait, we are supplized to find that any person walks almost in the same gait pattern. How do we select one pattern among so many adequate patterns? This proposition is called inverse kinetics. And also any normal human walk at almost the same speed. How come is it? And there are many ways of muscle motion to produce one walking gait pattern. How do we select one pattern of muscle motion among so many ways of motion? This proposition is called inverse dynamics. To solve the upper limb motion, some approaches have been proposed and obtained good results for this proposition, such as minimum jerk model or minimum torque-change model.

For the bipedal locomotion, which requires total body movement and highly complexed dynamic motion with problems of body form, inertia and the biarticular muscles etc., the approach may not be easy. Though, we hope it will be solved in near future. To solve this, we might require to measure the joint motion during walking, to analyze the mechanical energy flow between body segments, to measure the floor reaction force, to calculate the joint moments, to estimate the muscle activity, and to know the muscle dynamics and energy consumption characteristics of the muscle. Infant walking and geriatric walking should be also analyzed. EMG data and $\mathrm{O}_{2}$ consumpution data analysis are effectual means to check various assumptions. The computer simulation method will be able to tell the results when the body form is changed or the muscle origin and insertion is changed. With these analysis the process of the evolution and the advantageous of our bipedal locomotion will be revealed. Up to date informations will be shown in this seminar.

The bipedal locomotion has been developed as the style in which the body, especially the brain or other important internal organs, get the minimum impact, and muscles perform at their maximum efficiency and the body moves at their fastest speed in the given conditions or environments. When the gait style changes, the body form will be changed and vice versa. Bipedal locomotion of the human being have evolved in this complexity of the law of cause and effect. To analyze our gait is to look back to the past days and to recognize our own pass of evolution. Origin of our existence goes back to the big bang 10 billion years ago. 
Since the total energy in our universe is probably very constant, our energy of every steps must be from the big bang.

\section{H A L L B N G B $\quad$ T O}

Now let's look at the future. Bipedal locomotion has been produced in mechanical and physiological law of cause and effect, however, it is also affected by the psychological and social conditions. The modern man does not walk so long distance, and the problems which is peculiar to the erect walking is such as low back pain. There are many cases which disturb the normal bipedal locomotion, those are such as congenital malformation, diseases, war, traffic accidents, labor accidents, poison, damage from medicines and recently by sports injuries.

Many abnormal gait data have been accumulated by the effort of many pioneers in this field. And now we can calculate the quantitative score of the walking ability of the specific abnormal person in comparison with the normal person with a good reliability. The objective evaluation of gait is available with the automated gait measurement systems and computers. However this evaluation is the one which compares the abnormal person to the normal person. This is, so to speak, to evaluate the abnormal person with the methods which is developed for the evaluation of the normal gait. We desire to have the system, however, which can evaluate the abnormal person's ability in comparison with his potential ability.

The development of this kind of system seems to be impossibly difficult, and is not done so far. The clinician does not look at the patient's walking status as a third person when he observes his patient's gait with his own eyes. He looks the gait when he thinks that patient should improve this much under this kind of treatment. Observing and judging are done simultaneously. He eagers to have the data which can support his judgment. The data which merely shows the patient's present gait status has a little help, because to support his judgment, in this case, it requires the data which shows patients future gait status.

To challenge this situation we need to know the human normal gait mechanics, to know the optimum adaptation mechanisms in which the patients compensate their defect in a given condition. We also need to solve the inverse kinetics and inverse dynamics and to experiment the gait simulation. Statistical analysis to know the maximum gait ability of various patients with many kinds of functional impairments is also required. World wide compatible data sets should have important role for this purpose.

To accumulate and analyze the gait data it should be taken into account that the bipedal locomotion are influenced by the psychological and social conditions. Many patients feels such shocks as even not being able to stand up again after they become handicapped. Some other patients does not accept themselves to be 
handicapped, and keep telling that they shall be normal again and in turn they get strong stress from the gap between their wish and the reality. In both case, correct evaluation of their potential ability might be difficult.

Wisdom is required very much as well as the knowledge. This wisdom may be cultivated only in the process of the co-operation of engineers and medical personnel. Not only the partial charge of medical personnel and engineers for their role, but also some partnership more than collaboration are inevitable. Engineers should consider the patient's treatment and improvement, and medical personnel should think the treatment and the evaluation system on the general consideration not only their own patients. We should discuss on this seminar how can we evaluate patient's potential walking ability.

The patient's potential ability depends on what kind of means we have for the treatment, training and functional substitutions. So we should know the exact characteristics of these means. On this seminar we will pick up prostheses, orthoses and functional electric stimulators among these means, and examine the relation of these characteristics and the dynamics of the bipedal walking. Not to express the abnormality as merely the phenomenon but to think the way how we can improve their gait is our purpose on this seminar.

The gait analysis for the rehabilitation should be more aggressive. I think the term gait analysis overemphasize "analysis" and not adequate to express our challenging mind of helping patients to improve their gait. I would like to propose the new term "Gait Engineering". In this term the mutual deep collaboration between medical personnel and engineers are expected. I would be very happy if this seminar becomes the first step of the Gait Engineering.

\section{R B F P B R B N C B S}

1) (In Japanese) Imanami,Y.:Chemical study of the life, P3, Kodansya, (1980)

2) (Jap) Watanabe, S.:The life and the freedom, 128-130, Iwanami, (1980)

3) (Jap) Kagawa, Y. et al:The life and the energy, Baifukan, (1980)

4) (Jap) Nakamura, R., H. Saito:Basic kinesiology, Ishiyaku, (1987)

5) (Jap) Oparin, A. :The origin of the life and bio-chemistry, Iwanami, (1965)

6) (Jap) Schrödinger, E. : What is life ?, Iwanami, (1973)

7) (Jap) Miyashita, M.:Review of the gait analysis, Taiiku no kagaku, 15, 264-273, (1965)

8) Weber, W. \& E. Weber:Mechanik des menschlichen Gehwerkzeuge, (1836)

9) Saunders, J., V.T. Inman, H. D. Eberhart:The major determinants in normal and pathological gait, J Bone Joint Surg, 35A(3), 543-558, (1953)

10) (Jap) Kawato, M. :Computational neuroscience, BME, 3(10), 18-26, (1989) 